\title{
Benign Exocrine Pancreas Neoplasm
}

National Cancer Institute

\section{Source}

National Cancer Institute. Benign Exocrine Pancreas Neoplasm. NCI Thesaurus. Code C4613.

A benign, non-metastasizing neoplasm arising from the exocrine pancreas. 